\title{
Development of magnetic B-H hysteresis loops through stages of microstructure evolution of bulk BaFe12O19
}

\begin{abstract}
A series of polycrystalline $\mathrm{BaFe} 12 \mathrm{O} 19$ bulk samples produced have been used to investigate the parallel evolving relationship between the microstructural and magnetic properties of the magnetic material. The raw material starting powders were prepared via the mechanical alloying method and subsequently moulded and pressed by using a hydraulic pressing machine. The nanosized samples were sintered from 700 to $1300 \circ \mathrm{C}$ with $100 \circ \mathrm{C}$ increments in static air conditions. The sintered samples were characterized and analysed with increasing sintering temperature to study their crystallinity, microstructural and magnetic properties. The result shows the magnetic $\mathrm{B}-\mathrm{H}$ hysteresis loop varies with grain size, which was attributed to the increasing crystallinity and changing magnetic parameters. The microstructural properties like grain size were measured by using FeSEM, the phase analysis of the material was analysed with X-ray diffractometry (XRD) and density was determined by a densimeter, while the hysteresis loop was measured by a B-H tracer and Curie temperature was measured with an impedance analyser. The characterized samples were found to be divided into three groups which consisted of grain size varied from 0.30 to $0.39 \mathrm{\varepsilon m}$ (paramagnetic state), 0.46 to $0.94 \mathrm{\varepsilon m}$ (intermediate ferromagnetic state) and Ól.78 عm (strong ferromagnetic state). For the latter two groups, slanting sigmoid hysteresis loops were observed. Well-defined sigmoid-shaped B-H hysteresis loops were obtained when high crystallinity was attained, allowing strong exchange interaction between neighbouring atomic magnetic moments. This indicated that the ferromagnetic state had been achieved. The observed three B-H hysteresis groups exhibited different magnetic properties, and these behaviours are useful for industrial applications.
\end{abstract}

Keyword: Magnetic material; Magnetic properties; Microstructures 